University of Nebraska - Lincoln

DigitalCommons@University of Nebraska - Lincoln

Donald F. Easterling

SAIC, Brook Park, Ohio

Edward S. Hovanitz

SAIC, Brook Park, Ohio

Kenneth W. Street

NASA Glenn Research Center, Cleveland, Ohio

Follow this and additional works at: http://digitalcommons.unl.edu/nasapub

Easterling, Donald F.; Hovanitz, Edward S.; and Street, Kenneth W., "COMPARISON OF THE ADSORBED MERCURY SCREENING METHOD WITH COLD-VAPOR ATOMIC ABSORPTION SPECTROPHOTOMETRY FOR DETERMINATION OF MERCURY IN SOIL" (2000). NASA Publications. 173.

http://digitalcommons.unl.edu/nasapub/173 


\section{COMPARISON OF THE ADSORBED MERCURY SCREENING METHOD WITH COLD-VAPOR ATOMIC ABSORPTION SPECTROPHOTOMETRY FOR DETERMINATION OF MERCURY IN SOIL}

Keywords: Mercury, Cold Vapor Atomic Absorption, Mercury Vapor Analyzer, Soil Samples, Gold Films, Jerome Gold-Film Detection

Donald F. Easterling and Edward S. Hovanitz, SAIC (and formerly of Bionetics Corp.), 2001 Aerospace Parkway, Brook Park, Ohio. Kenneth W. Street, NASA Glenn Research Center, MS 23-2 21000 Brookpark Road, Cleveland, Ohio 44135.

\section{ABSTRACT}

A field screening method for the determination of elemental mercury in environmental soil samples involves the thermal desorption of the mercury from the sample onto gold and then the thermal desorption from the gold to a goldfilm mercury vapor analyzer. This field screening method contains a large number of conditions that could be optimized for the various types of soils encountered. In this study, the conditions were optimized for the determination

*To whom correspondence should be addressed. 
of mercury in silty clay materials, and the results were comparable to the coldvapor atomic absorption spectrophotometric method of determination. This paper discusses the benefits and disadvantages of employing the field screening method and provides the sequence of conditions that must be optimized to employ this method of determination on other soil types.

\section{INTRODUCTION}

The determination of mercury in environmental soil samples is complicated by the matrix and the need for analytical instrumentation capable of detecting very low concentrations. As a consequence, the methodology that is accepted by most organizations interested in environmental soil analysis usually involves two steps, separation of the mercury from the matrix and determination of concentration by a spectroscopic method. Of the spectroscopic techniques, the most frequently employed involve cold-vapor atomic absorption spectroscopy (CV-AA) or cold-vapor atomic fluorescence spectroscopy (CV-AFS). To enhance detectability, many methods have been modified after extraction to sweep into a concentrating apparatus the elemental mercury formed during reduction. The apparatus typically used for the concentrating is a thin layer of gold coating on a variety of substrates, such as gold-coated sand in a column. ${ }^{1-6}$ The elemental mercury is then removed by flash heating the gold-lined tube with a slow flow of a carrier gas, which deamalgamates the mercury into the detection instrument. This field screening method (FSM) is a combination of previously evaluated gold sorption technologies and is also referred to as the adsorbed mercury analysis method. Widely used in the mineral exploration industry for many years, it measures small differences in mercury concentrations in surface soils, thus providing a quick and inexpensive means to locate ore deposits buried well below the Earth's surface. It was also applied as a general screening method for environmental investigations that the NASA Glenn Research Center (GRC) conducted under an agreement with the Ohio Environmental Protection Agency. The method proved to be useful in detecting mercury in soil, but it appeared to 
produce somewhat erratic results with an unexpected positive bias. When comparing it with the traditional and well-accepted CV-AA method, which sees all forms of mercury, one would have expected the FSM to have a negative bias since it does not detect all mercury species and is not likely to detect mercury incorporated in the soil mineral structure. ${ }^{7-9}$

The field screening method requires no digestion of soil samples; sample preparation is limited to air-drying and sieving. Soil samples are heated in goldlined tubes to drive the mercury into the gold. Subsequently, the gold-lined tubes are heated above the deamalgamation temperature to liberate the mercury, which is drawn into the Jerome, a gold-film mercury vapor analyzer. It is used as an inexpensive and very sensitive alternative to spectroscopy arid functions by adsorbing elemental mercury from the gas phase directly onto a gold film and subsequently determining the concentration by the change in electrical resistance to the gold film. Thus, concentration and determination are combined in one step. In environmental soil samples, the mercury is frequently in the elemental state, making the use of the gold sorption mechanism a viable altemative for sample processing, especially for screening purposes. ${ }^{10}$

This study was designed to define the performance of the FSM by comparison with the CV-AA and to explain the unexpected positive bias. Two well-defined soil samples having significantly different mercury concentrations were collected, homogenized, and characterized for this investigation. In addition to comparing the results for the two methods, the field screening method conditions were optimized. The optimized conditions were significantly different from the conditions employed in the original FSM data from the GRC study and may have contributed to the difference in results obtained from the two methods.

\section{EXPERIMENTAL}

\section{Instrumentation}

The adsorbed mercury analysis method (FSM) uses an initial heating unit 
for soil incubation, a final heating unit for mercury recovery, and a mercury vapor detector. The initial heating unit (Fig. 1(a)) consists of a Thermodyne type 1900 hot plate that heats a 15 - by $15-$ by $6.4-\mathrm{cm}$ aluminum block having five rows of five holes spaced $25.4 \mathrm{~mm}$ apart and centered in the block. Each hole is $8 \mathrm{~mm}$ in diameter and $40 \mathrm{~mm}$ deep. The quartz sample tubes, $6 \mathrm{~mm}$ in diameter and $60 \mathrm{~mm}$ in length, are coated on the inside bottom $(30 \pm 2 \mathrm{~mm})$ with a thin layer of gold. Temperature is measured using a Digi-Sense type $\mathrm{K}$ thermocouple thermometer. The final heating unit (Fig. (1(b)) consists of a resistance coil flash heater, a variable ac power source (Staco Energy Products Co., type 3PN1010 Variable Autotransformer; i.e., a standard variac), a thermocoüple, a purging assembly, and a charcoal filter. The purging assembly consists of $1.6-\mathrm{mm}-\mathrm{od}$, stainless steel inlet and outlet tubing and a thermocouple imbedded in a composite purging head. The filter is constructed by filling a $15-\mathrm{mm}$-id plastic tube with a $100-\mathrm{mm}$ length of granular carbon media. These components are connected with Eastman Nulo-Seal tubing (2-mm id). The initial and final heating units were obtained from Microseeps, the University of Pittsburgh Applied Research Laboratory. The detector, a Jerome model 411 gold-film mercury vapor analyzer, was obtained from the Arizona Instrument Corporation and was operated and calibrated according to the manufacturer's specifications.

A computer-controlled Perkin Elmer model FIAS 100 cold-vapor atomic absorption spectrometer, equipped with a Perkin Elmer model AS 90 autosampler and operated and calibrated according to manufacturer's specifications, was used for CV-AA determinations.

\section{CV-AA Reagents}

The SW846 method 7471A was followed without exception. " All reagents used in the study were reagent grade from Fisher Scientific. The chemicals utilized for the study were stannous chloride, hydroxylamine hydrochloride, concentrated hydrochloric acid, concentrated nitric acid, sulfuric acid, sodium 

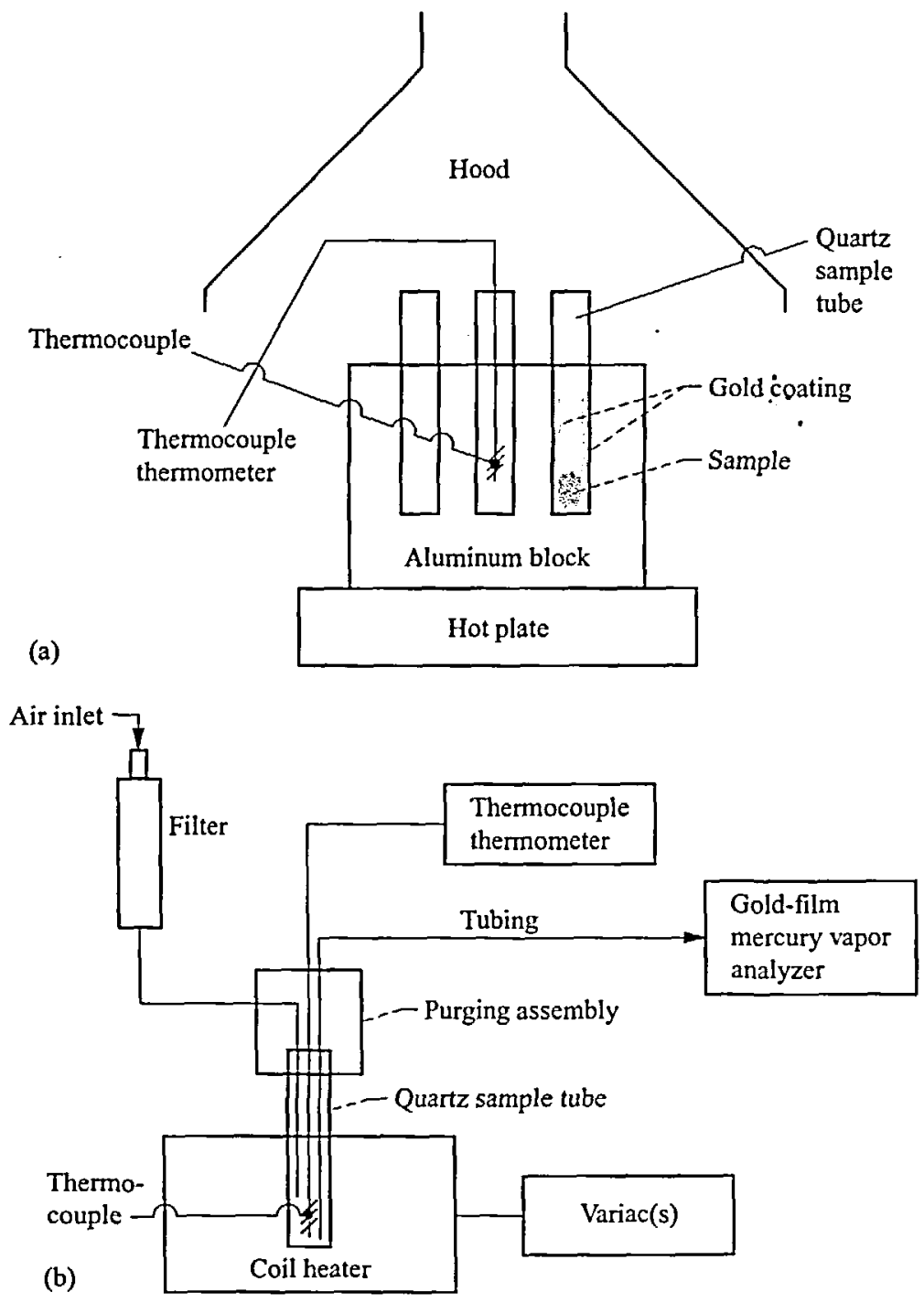

Fig. 1: Instrumentation for adsorbed mercury analysis method (FSM). (a) Initial heating unit. (b) Final heating unit. 
chloride, and potassium permanganate. All reagents were prepared according to the method, and deionized water was used throughout.

\section{Standards}

The Jerome calibration standard was a saturated mercury vapor prepared by placing approximately $20 \mathrm{~g}$ of elemental mercury in a 200-ml sealed Pyrex jar and maintaining it at a constant temperature of $21.5 \pm 0.5^{\circ} \mathrm{C}$ in a Polyscience model 9005 circulating water bath in accordance with the manufacturer's instructions. The Jerome calibration was performed prior to use by the injection of $1.0 \mathrm{ml}$ of the saturated mercury vapor that contained $16,3 \mathrm{ng}$ mercury (calculated from the manufacturer's calibration literature). The CV-AA mercury calibration standards were prepared by dilution from a SpecCertiPrep 1000-ppm mercury in 10-percent nitric acid reagent. A 1-ppm stock standard was prepared fresh daily by serial dilution with 0.5 -percent nitric acid. Working standards of 5 and $10 \mathrm{ppb}$ were prepared fresh daily by the dilution of the 1.0-ppm stock standard with 0.5-percent nitric acid.

\section{Soil Standards}

Commercially available soil standards were obtained from the National Institute of Standards and Technology, NIST, (SRM 8407 containing $50 \mu \mathrm{g} / \mathrm{g}$ mercury) and from Environmental Resources Associates, ERA, (Priority PollutnTM/CLP, lot 229 containing $1.5 \mathrm{mg} / \mathrm{kg}$ mercury). Commercial soil standards were used as received.

Three wet samples (several $\mathrm{kg}$ ) of soil were collected from three locations at the NASA Glenn Research Center and were placed in large plastic bags. These samples were spread out on plastic in a hood and were air dried at room temperature for 3 weeks. It was assumed that little elemental mercury is lost under these conditions. ${ }^{7,10}$

Rocks, twigs, and other foreign objects were removed from each sample. The soil, primarily a light yellow silty clay, was then blended by repetitive 
alternate shoveling. After the initial blending, it was gently broken up in a mortar and passed through a 10-mesh screen. It was then subjected to additional homogenization, but only the portion passing the 10 -mesh screen $(<2.0-\mathrm{mm}$ diam) was utilized. An $x, y$-grid was constructed over the spreadout sample from which scoops were taken in order from each grid location. The process was repeated using the exact sample sequence until all the sample was collected in a single container. This portion of the sample was labeled "10-mesh" material and was used for CV-AA characterization and as noted elsewhere. A large portion of the 10-mesh material was mortared and sieved through a 40-mesh screen. Each fraction was collected and labeled as $>40$ mesh ( $<0.43-\mathrm{mm}$ diam). or $<40$ mesh (>0.43-mm diam) for analysis. Each sample was labeled and stored in a Tefloncapped glass container for subsequent use. These samples were extensively characterized by CV-AA and two of the three soil samples were used as reference standards (designated $\mathrm{L}$ and $\mathrm{H}$ ) for optimization and method comparison.

\section{Procedure for CV-AA}

Samples requiring CV-AA analysis were prepared and analyzed according to SW846 method 7471A. " This method requires the digestion of samples using aqua regia and potassium permanganate, the reduction of excess oxidant with hydroxylamine, the reduction of the mercury to elemental state with stannous chloride, and quantification using cold-vapor atomic absorption spectrophotometry. Calibration of the unit was accomplished with a blank 5- and 10 -ppb standard. Soil samples were determined from the plot of the calibration standard signal versus the concentration.

\section{Procedure for Adsorbed Mercury Analysis Method (FSM)}

Two variations of this method were used, the industrial and the optimizedconditions. The industrial method appears first followed by the optimizedconditions in parentheses. 
To prepare the sample, the soil is spread out on a clean, disposable plastic surface and is air-dried overnight at room temperature in a laboratory hood. It is then gently broken up and sieved through a 40 -mesh screen. Only the portion passing through the screen is utilized.

Each gold-coated tube is numbered and the exterior top portion wrapped with Teflon tape to provide an airtight seal during the final heating stage. Before use, each sample tube is cleaned by placing it in the flash heater and heating to $550{ }^{\circ} \mathrm{C}$ until three successive measurements on the Jerome indicate that no mercury is coming off the tube. To reduce the potential for adsorption of mercury from ambient air during storage, the tube is immediately. sealed with an airtight plastic cap. Since only the bottom of each tube was heated, the tops remained relatively cool so as to allow for immediate capping.

The aluminum block is placed on top of the hot plate and is heated under a laboratory hood until a constant temperature of $175^{\circ} \mathrm{C}\left(225^{\circ} \mathrm{C}\right)$ is achieved. The temperature is determined by placing the type $\mathrm{K}$ thermocouple into an empty tube in the center of the heating block. A 0.2- to 0.5- (0.05- to 0.2-) gram sample is weighed using a four-place analytical balance. The lesser amount of soil is used when high concentrations of mercury are expected in order to prevent saturation of the Jerome gold-film detector. The sample number, tube number, and sample weight are recorded, and the sample is placed in the tube. The tubes containing samples are placed in the aluminum block heater and heated for $1 \mathrm{hr}$ at $175^{\circ} \mathrm{C}\left(225^{\circ} \mathrm{C}\right)$. The tubes are immediately removed from the heating block and cooled. The soil is removed by shaking. Any residual soil is removed by blowing grease-free compressed air into the tube.

Sample determination is initiated in the final heating unit. The Jerome is calibrated with the mercury vapor standard prior to taking sample readings. The sample tube is seated in the purging assembly and then inserted into the resistance coil flash heater. The flash heater is switched on and the temperature is increased using the variac until the temperature reaches $550^{\circ} \mathrm{C}$. At this point, the Jerome 10-sec cycle is initiated, and the cycling is repeated until no 
additional mercury is detected. Care must be taken to ensure that the time elapsed from flash initiation to the Jerome sample reading remains fairly constant. The individual meter readings are recorded and added together for individual samples.

The concentration of mercury $(\mathrm{Hg})$ in the soil, expressed as $\mathrm{mg} / \mathrm{kg}(\mathrm{ppm})$, uses the ratio of the reading for the mercury vapor standard to that of the soil:

$$
\mathrm{mg} / \mathrm{Kg} \mathrm{Hg}=\frac{\text { (Sample Jerome meter reading)(ng Hg in standard) }}{\text { (Standard Jerome meter reading)(mg sample) }}
$$

\section{RESULTS AND DISCUSSION}

The NASA Glenn Research Center in Cleveland, Ohio, has done an extensive characterization of soil for potential mercury contamination. Because of the expense involved and the number of samples required to complete the preliminary evaluation, a group of samples was submitted for CV-AA and an extensive number of samples was submitted for characterization by the FSM. A poor correlation was obtained for the samples determined by both methods (see Fig. 2). The FSM uses the gold extraction of mercury from soil and the gold-film mercury vapor analyzer, both of which have been demonstrated to be quantitative. Therefore, it should be straightforward to combine the two technologies into a quantitative method. The wide scatter in data between the two prompted us to investigate the source of discrepancy in the CV-AA and the field screening methods. Obtaining reliable, quantitative data from the FSM is attributed to the advantages of this method; it is simpler, faster, and does not rely on reagents that introduce further waste disposal issues. Therefore, we investigated possible sources of error associated with the field screening method and in the process have optimized several conditions associated with sample treatment and the analytical measurement steps. 


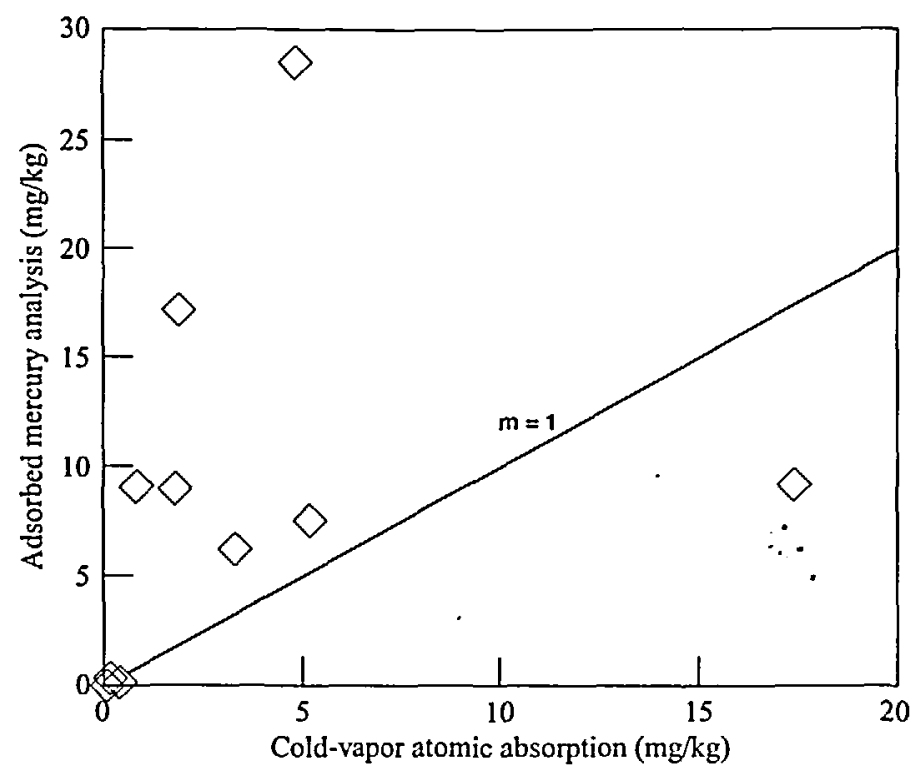

Fig. 2: Comparison of initial sample determinations made by adsorbed mercury analysis method (FSM) and by cold-vapor atomic absorption method (CV-AA).

\section{Sample Preparation}

To compare the two analytical methods, the samples were homogenized and sieved to provide a sample that was nearly identical for both methods. Note, however, that the CV-AA method allows for larger samples and larger particle sizes because the FSM is limited by the amount of sample that can be conveniently processed in the small, gold-coated quartz tubes (these samples will not contain high enough levels of mercury to saturate the detection system of the Jerome; saturation of the Jerome occurs after a total accumulation of 0.5 $\mu \mathrm{g}$ of mercury in the gold film. For samples containing low levels of mercury, a large number of samples can be determined prior to saturating the film.

To determine whether the sources of error with the FSM were due to the sampling or to the handling of the sample prior to sample extraction, the matrix 
of experiments that was performed included varying the sample weight and the particle size. Tables 1 and 2 give the results of the tests performed. Unless otherwise specified, the FSM procedure employed is the standard one provided by the firm that performed the determinations used to construct Figure 2.

Table 1 presents the results in terms of data variability as a function of sample size. It appears that there is no improvement in either the signal or standard deviation for a homogeneous sample by varying sample size. Table 2 presents the results in terms of the data variability as a function of particle size. It is apparent that the larger size particles lead to greater variability in the determined values and lower concentrations of mercury. It is likely that the larger particle material contains larger grain materials with less surface area to adsorb mercury. Better correlation is seen between CV-AA and the FSM for unseived materials; however, in practice, the CV-AA method calls for 10-mesh material and the FSM relies on small-particle material because of the limitations imposed by the size of the gold sample tubes and the need to more efficiently pack them. With respect to the FSM, note also that the aggregation of smaller particles into larger masses could potentially impede the transfer of mercury to the gold collection surface.

Figure 3 is an evaluation of the initial heating temperature for the process of driving the mercury out of the soil and into the gold collection tube. It is very apparent that there is an optimal temperature. At low temperatures, the mercury is not fully volatilized for transport to the gold. At higher temperatures, the gold does not effectively accumulate the mercury. This latter observation correlates well with the deamalgamation temperature for mercury-gold systems reported to be around $280{ }^{\circ} \mathrm{C}^{8}$ As the deamalgamation temperature is reached, the equilibrium that is established is more favorable for the mercury to remain in the vapor over the gold; thus, when the incubation is completed, the mercury is either lost in the gas above the sample or reabsorbed back into the sample. The optimized temperature for collection of the mercury is between 200 and $225^{\circ} \mathrm{C}$, which gives considerably higher recovery for this type of sample than the $175^{\circ} \mathrm{C}$ 
TABLE 1

Variability of Data as Function of Sample Size for Unoptimized Adsorbed Mercury Screening Method (FSM) and Cold-Vapor Atomic Absorption Method (CV-AA)

\begin{tabular}{|c|c|c|c|c|c|}
\hline \multirow[t]{3}{*}{ Sample } & \multirow[t]{3}{*}{$\begin{array}{l}\text { Weight, } \\
\text { mg }\end{array}$} & \multicolumn{4}{|c|}{$\begin{array}{c}\text { Mercury determined, } \\
\mathrm{mg} / \mathrm{kg}\end{array}$} \\
\hline & & \multicolumn{2}{|r|}{ FSM } & \multicolumn{2}{|c|}{ CV-AA } \\
\hline & & Average & $\begin{array}{l}\text { Standard } \\
\text { deviation }^{\mathrm{a}}\end{array}$ & Average & $\begin{array}{l}\text { Standard } \\
\text { deviation }^{\mathrm{a}}\end{array}$ \\
\hline $\mathrm{L}$ & $\begin{array}{l}100 \\
250 \\
500 \\
\end{array}$ & $\begin{array}{l}0.042 \\
0.046 \\
0.033 \\
\end{array}$ & $\begin{array}{l}0.011(10) \\
0.009(15) \\
0.010(9) \\
\end{array}$ & 0.194 & $0.015(6)$ \\
\hline $\mathrm{H}$ & $\begin{array}{l}50 \\
100 \\
150 \\
\end{array}$ & $\begin{array}{l}0.399 \\
0.398 \\
0.408 \\
\end{array}$ & $\begin{array}{l}0.075(6) \\
0.042(10) \\
0.035(6) \\
\end{array}$ & 0.910 & $0.042(4)$ \\
\hline
\end{tabular}

${ }^{\mathrm{a}}$ Parentheses contain the number of samples.

TABLE 2

Variability of Data as Function of Particle Size for Unoptimized Adsorbed Mercury Screening Method (FSM) and Cold-Vapor Atomic Absorption Method (CV-AA)

\begin{tabular}{|c|l|l|l|l|l|l|}
\hline \multirow{2}{*}{$\begin{array}{l}\text { Sample } \\
\text { H }\end{array}$} & \multicolumn{5}{|c|}{ Mercury determined, mg/kg } \\
\cline { 2 - 7 } mesh size & $\begin{array}{l}\text { Sample } \\
\text { weight, } \\
\text { mg }\end{array}$ & Average & $\begin{array}{l}\text { Standard } \\
\text { deviation }\end{array}$ & $\begin{array}{l}\text { Sample } \\
\text { weight, } \\
\text { mg }\end{array}$ & Average & $\begin{array}{l}\text { Standard } \\
\text { deviation }\end{array}$ \\
\hline 10 & 50 & 0.77 & $0.07(13)$ & 500 & 0.82 & $0.05(6)$ \\
$<40$ & 100 & 0.64 & $0.07(13)$ & 530 & 0.91 & $0.04(4)$ \\
$>40$ & 150 & 0.28 & $0.05(13)$ & 520 & 0.50 & $0.21(4)$ \\
\hline
\end{tabular}

${ }^{3}$ Parentheses contain the number of samples.

initial temperature specified in the original procedure (before the FSM was optimized). This parameter must be evaluated if the method is to be performed with other types of materials such as loam soils.

The initial heating time for the incubation of the samples was also examined. The time required is a function of the incubation temperature; the higher the temperature, the faster the desorption/amalgamation occurs. Figure 4 


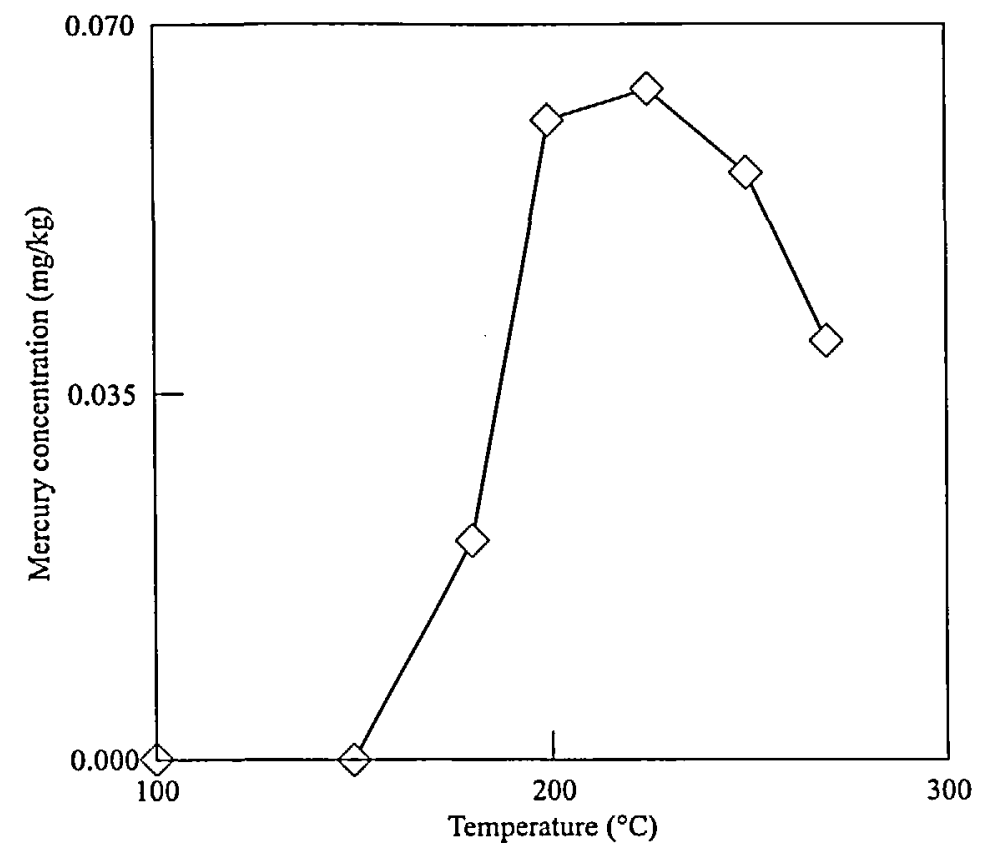

Fig. 3: Mercury recovery from sample as a function of initial heating temperature.

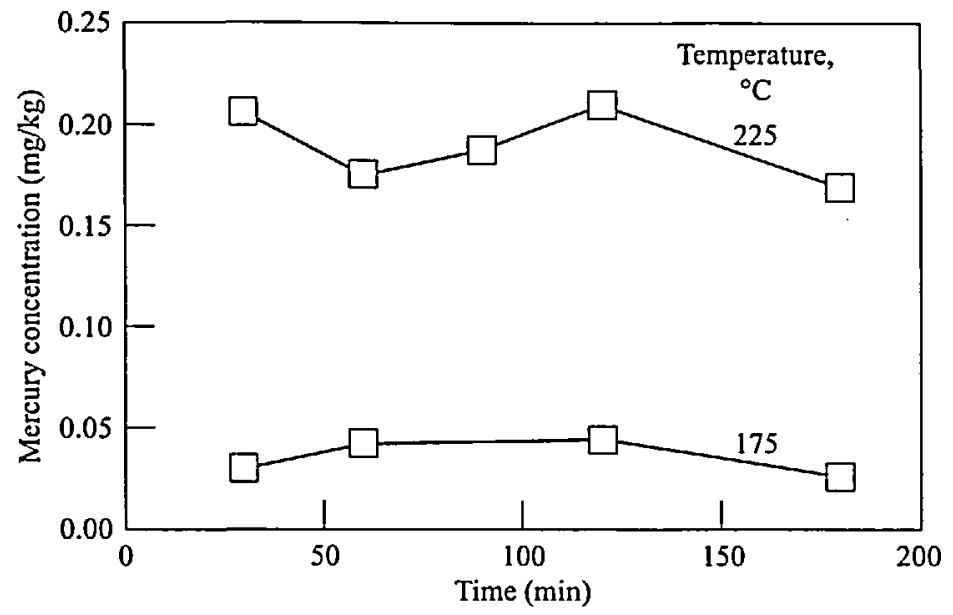

Fig. 4: Mercury recovery from sample as a function of initial heating time at temperatures of 175 and $225^{\circ} \mathrm{C}$. 
makes it apparent that the minimum time required to extract the mercury from this type of soil at $175^{\circ} \mathrm{C}$ is $1 \mathrm{hr}$ (according to the original procedure) and that better recoveries are possible for longer incubation times. The figure also shows that at the optimum desorption temperature of $225^{\circ} \mathrm{C}$, the recovery of mercury from the samples is nearly doubled for even $30 \mathrm{~min}$ of incubation and that it may be possible to incubate efficiently for even shorter periods. At a $225{ }^{\circ} \mathrm{C}$ incubation, the results from the FSM determinations approach the values obtained from the parallel CV-AA determinations. This parameter must be evaluated if the method is to be performed with other types of materials such as loam soils.

It is unlikely that cross-contamination of the samples will occur during the incubation of samples in a well-ventilated hood. A sample tube containing 250 $\mathrm{mg}$ of the 50-ppm NIST standard was placed in the center of four empty sample tubes and incubated according to the original procedure. The blank tubes had zero readings, as anticipated. In terms of laboratory cross-contamination of samples, blank tubes were tested with the hood door open, allowing laboratory air to pass directly over the tops of the sample tubes, and with the hood door closed, causing the circulation to travel up towards the tops of the tubes from the base of the hot plate used for maintaining the block temperature. All these tubes read zero.

As an aside, it has been our experience that the incubation block requires several hours to achieve a stable and constant temperature in the current configuration. For routine processing of samples, it would be more economical to develop a fixed block device rather than to rely on the aluminum mass to distribute the temperature evenly. Even in the current configuration, situated in the draft of a fume hood, the central holes in the block maintained a moderately even and reproducible temperature. In an incubation block designed specifically for this type of analysis, it should be possible to process at least 100 samples simultaneously. After the mercury is collected in the gold sample tubes and the 
sample is removed, the tubes appear to be stable for over a week, although the maximal storage time was never determined. ${ }^{9}$

\section{Sample Desorption}

It is necessary to use a flash temperature in excess of the deamalgamation temperature $\left(280^{\circ} \mathrm{C}\right)$ to drive the collected mercury from the gold. Figure 5 is a plot of the ratio of the second Jerome reading during a sample desorption (see subsequent paragraph for further detail) to the total reading versus the temperature of the desorption. This figure demonstrates that little optimization is gained by using flash temperatures other than that provided by. the original method $\left(550^{\circ} \mathrm{C}\right)$. The optimized deamalgamation process for films, sand traps, coated tubes, and so forth has never been determined because it appears to be a function of the device, its geometry, the gold thickness, and the experiment in which it is used. The literature reports adequate desorption at temperatures as low as $300{ }^{\circ} \mathrm{C}$ and incomplete desorption for temperatures as high as $450^{\circ} \mathrm{C}^{2}$ (this same paper then presents a figure that appears to have adequate desorption at $400{ }^{\circ} \mathrm{C}$ ). Other reports use desorption temperatures ranging from 500 to 800 ${ }^{\circ} \mathrm{C} .3,5,6$

Not all the mercury is desorbed during the first reading of the flash heating step. A significant amount of mercury is detected during the second and often the third Jerome readings of a single thermal flash vaporization of a mercurycontaining sample tube. During $10 \mathrm{sec}$ of unrestricted flow, the Jerome samples $125 \mathrm{ml}$ of gas. The calculation of the volume of gas sampled versus the volume of gas in the system between the gold tube and the detector film indicates that one sample reading should be more than sufficient to collect all the mercury. The transfer tubing between the sample tube and the detector has a small diameter so as to maintain a small transfer volume and to assist in the complete transfer of the sample volume to the detector. However, this tubing also acts as a flow restrictor and the heating of the sample tube further reduces the flow rate (see Table 3). Even at these flow rates, the sampling volume should be more than 


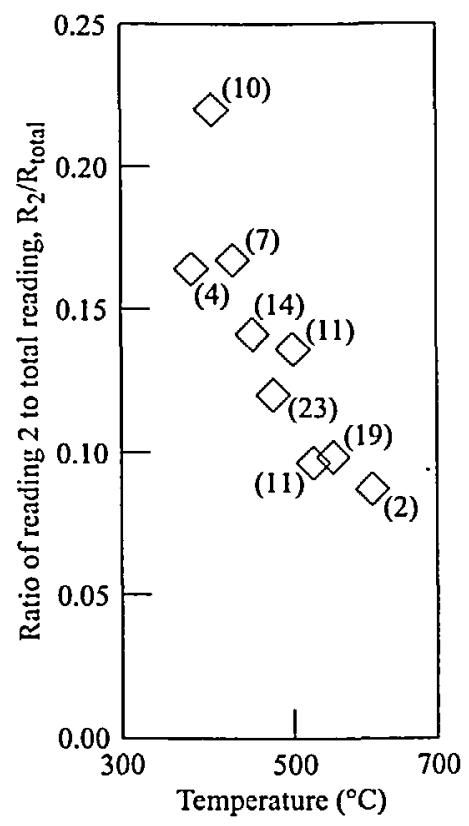

Fig. 5: Ratio of second meter reading to total meter reading as function of desorption temperature. Data weighted by number of samples in subsets, and number in parentheses is total number of samples determined.

TABLE 3

Flow Rate Entering Gold-Film Mercury Vapor Analyzer

\begin{tabular}{|l|l|c|}
\hline Conditions & $\begin{array}{l}\text { Flash heater } \\
\text { temperature, }{ }^{\circ} \mathrm{C}\end{array}$ & $\begin{array}{l}\text { Flow rate, } \\
\text { ml/min }\end{array}$ \\
\hline Direct to detector & 23 & 13.4 \\
& 23 & 5.1 \\
& 280 & 5.2 \\
& 400 & 4.5 \\
& 500 & 4.2 \\
& 550 & 4.1 \\
& 600 & 4.0 \\
\hline
\end{tabular}

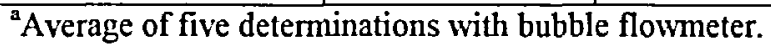


sufficient to sweep all vapor-phase mercury into the Jerome to be detected within one sample reading. This hypothesis was confirmed by injecting mercury calibration gas into various points in the sampling train where an uncoated quartz tube replaced the gold-coated sample tube and then taking sample readings (Table 4 ).

One explanation for the high amount of mercury in readings subsequent to the first one following the flash is that there is a minimum time necessary to desorb the mercury from the gold sample tube. Several references address this issue and conclude that the gold should be on the order of less than 1 micron in thickness to eliminate memory effects and achieve complete desorption in a realistic amount of time. The mercury is known to amalgamate rather than surface sorb and hence the desorption process is diffusion limited.

As the temperature is not the only determining factor, desorption time was evaluated. The desorption time was extended by using a double-variac method in which the first variac was set to yield a flash desorption temperature approximately 1.5 to 2 times the desired temperature. The second variac was connected to the first variac and was used to adjust the temperature (analogous to coarse and fine adjustments). Figure 6 demonstrates the effect of using the second variac to adjust temperature. The crossover point of the two curves is approximately at the deamalgamation temperature for mercury; hence, the time for deamalgamation is approximately from this point to where the first reading of mercury occurs (marked by arrows on the curves). The double-variac method approximately doubles the time for deamalgamation but provides no improvement in the number of readings required, standard deviation for sample determinations, or control over final desorption temperature $\left(0.99_{ \pm} 0.17 \mathrm{mg} / \mathrm{kg}\right.$ $(n=9)$ for the single-variac study versus $0.96 \pm 0.13 \mathrm{mg} / \mathrm{kg}(\mathrm{n}=10)$ for the double-variac study on 50-mg samples of the $\mathrm{H}$ standard). By simple inspection of the gold tubes, it is apparent that the gold thickness is not homogeneous because of the manner in which the tubes are coated with gold by the 


\section{TABLE 4}

Gold-Film Mercury Vapor Analyzer Meter Reading Associated With Location of Standard Injection

\begin{tabular}{|l|l|c|}
\hline Injection location & $\begin{array}{l}\text { Flash temperature, } \\
{ }^{\circ} \mathrm{C}\end{array}$ & Meter reading \\
\hline Analyzer inlet (not & 23 & 0.093 \\
attached to flash & & \\
heater) & & 0.089 \\
Analyzer inlet & 23 & 0.099 \\
Analyzer inlet & 550 & 0.101 \\
Flash heater inlet & 23 & 0.100. \\
Flash heater inlet & 550 & \\
\hline
\end{tabular}

${ }^{a}$ Average of three to five injections of $16.4 \mathrm{ng}$ of mercury.

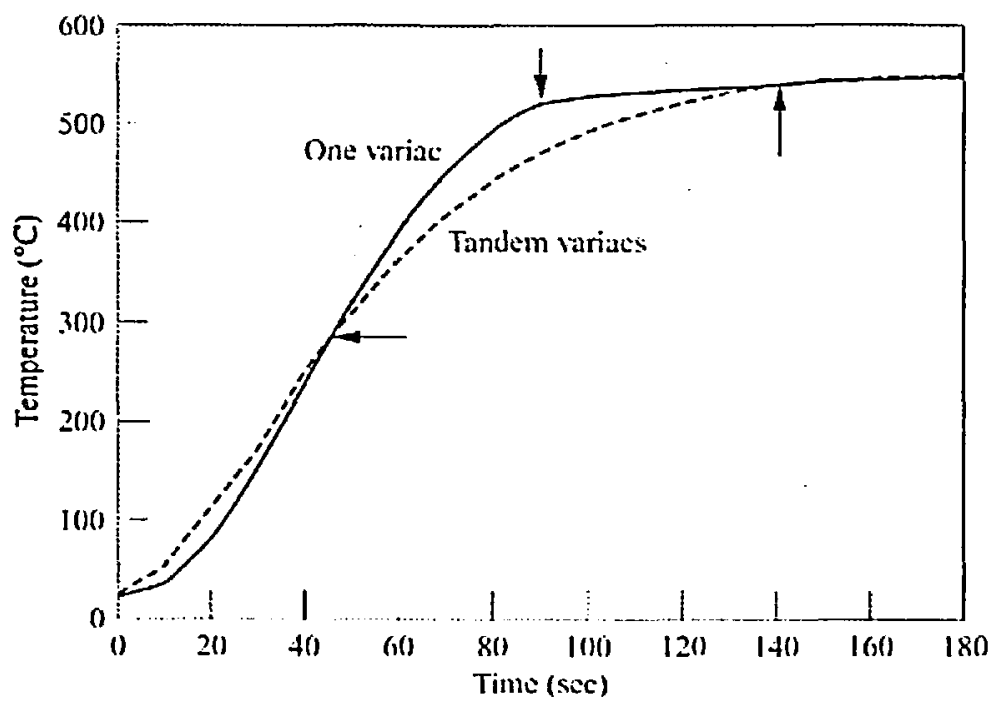

Fig. 6: Temperature inside sample tube as function of time for one variac and tandem variacs. Vertical arrows indicate approximate time of first meter reading, and horizontal arrow indicates approximate deamalgamation temperature of mercury from gold. 
manufacturer. It may be that if there were better quality control over the film thickness, some of these conditions could be optimized further.

The original reason for evaluating the double-variac method was to improve our ability to achieve constant desorption curves (as in fig. 6) for the method. In the single-variac method, small changes in the variac setting, which only uses 10 percent of the variac power, translate to large fluctuations in flash temperature when the flash is powered by using the power control rather than the on-off switch. In practice, using the on-off switch to flash the tubes provides a convenient means of achieving reproducibility after the variac has been adjusted to the correct power setting. (The directions provided with the method included flashing the tubes by adjusting the power setting which is not reproducible and requires an experienced analyst to make it work properly.) Finally, the use of a single variac places considerable strain on the few internal windings used to regulate the power delivered to the heater, a situation that can be remedied by using tandem variacs.

\section{Gold-Film Mercury Vapor Analyzer}

Because the Jerome is reported to be sensitive to sudden temperature changes, we placed a thermocouple in front of the Jerome inlet to determine if the temperature of the gold film was being elevated during the sampling. Also, our experience has been that the Jerome is very sensitive to changes in film temperature associated with recycling the film after saturation. A study that was performed varied the flash temperature from room temperature to $600^{\circ} \mathrm{C}$ while the temperature to the Jerome inlet was monitored. Over this temperature range, the inlet temperature remained at room temperature during the flash heating.

The instrument detection limit for mercury is $0.5 \mathrm{ng}$, which would yield a method detection limit of 1 to $10 \mu \mathrm{g} / \mathrm{kg}$ for samples of 500 to $50 \mathrm{mg}$, respectively. These calculated limits are in the range of mercury typically associated with background levels for uncontaminated soils. The background is associated with the high mobility of mercury in soil because of its volatility 
and/or the deposition of atmospheric mercury generated by the burning of coal. ${ }^{12}$ For example, typically contaminated soils have mercury concentrations an order of magnitude greater than these numbers (see details of NIST SRM 8407) These method detection limits are well below NASA Glenn's naturally occurring soil mercury concentrations of $50 \mu \mathrm{g} / \mathrm{kg}$.

The actual detection limits for the method are a function of the FSM variability to produce a minimal standard deviation for small and often inhomogeneous samples. The method detection limit is further subject to the performance characteristics of the Jerome analyzer. When the Jerome has a fully charged battery and freshly recycled detector film, the unit performs much more efficiently than when either/or both are partially used. In the best determination sets of this study using the optimized conditions, standard deviations on the order of $0.016 \mathrm{mg} / \mathrm{kg}$ for the $\mathrm{L}$ sample were obtained, which corresponds to a detection limit of $0.04 \mathrm{mg} / \mathrm{kg}$ at the 95 -percent confidence level. Background levels of mercury in soil are typically in the range of the method detection limit and therefore the FSM should be applicable to the detection of contamination levels above the background. ${ }^{13}$ Samples having a higher concentration (1-ppm level) deplete the film capacity quickly, or when too many samples are determined on the gold film, the standard deviations are typically $0.05 \mathrm{mg} / \mathrm{kg}$ or greater, corresponding to a method precision in excess of $0.1 \mathrm{mg} / \mathrm{kg}$ (RSD around 10 percent for determination).

Table 5 summarizes studies performed on the Glenn-generated soil standards. In general, the CV-AA results were elevated over the FSM and the optimized FSM. The elevated results could be explained by the fact that the CVAA method gives total mercury (elemental mercury plus mercury salts and organomercurials) and the FSM and optimized FSM only give total elemental mercury. By careful sampling and numerous studies not mentioned here, it is apparent that the negative bias of the FSM is because of incomplete recovery, a problem largely removed in the optimized FSM. Examination of the $\mathrm{H}$ soil sample indicated that the majority of the mercury resides in the finer particles. In 
TABLE 5

Comparison of Results From Unoptimized and Optimized Adsorbed Mercury

Screening Method (FSM) and Cold-Vapor Atomic Absorption (CV-AA)

\begin{tabular}{|c|c|c|c|c|c|c|c|}
\hline \multirow[t]{3}{*}{ Sample } & \multirow[t]{3}{*}{$\begin{array}{l}\text { Weight, } \\
\text { mg }\end{array}$} & \multicolumn{6}{|c|}{$\begin{array}{c}\text { Mercury determined, } \\
\mathrm{mg} / \mathrm{kg}\end{array}$} \\
\hline & & \multicolumn{2}{|c|}{ Unoptimized FSM } & \multicolumn{2}{|c|}{ Optimized FSM } & \multicolumn{2}{|c|}{ CV-AA } \\
\hline & & Mean & $\begin{array}{l}\text { Standard } \\
\text { deviation }^{\mathbf{a}}\end{array}$ & Mean & $\begin{array}{l}\text { Standard } \\
\text { deviation }\end{array}$ & Mean & $\begin{array}{l}\text { Standard } \\
\text { deviation }^{\mathrm{a}}\end{array}$ \\
\hline $\mathrm{L}$ & $\begin{array}{l}100 \\
250\end{array}$ & $\begin{array}{l}0.042 \\
0.046\end{array}$ & $\begin{array}{l}0.011(10) \\
0.009(15)\end{array}$ & 0.141 & $0.016(8)$ & 0.194 & $0.015(6)$ \\
\hline $\mathrm{H}$ & $\begin{array}{l}50 \\
530\end{array}$ & 0.640 & $0.074(13)$ & 0.936 & $0.144(8)$ & 0.910 & $0.042(4)$ \\
\hline
\end{tabular}

${ }^{\mathbf{a}}$ Parentheses contain the number of samples.

the case of the $\mathrm{H}$ and $\mathrm{L}$ samples, there is a good correlation between the CV-AA and the optimized FSM although the same cannot be said conceming the original FSM. The majority of the deviation between the methods appears to be due to the incubation temperature difference. Even using the unoptimized FSM, there should have been a much better correlation between the FSM and CV-AA methods employed for generating the data for Figure 2 with a negative bias for the FSM results. The lack of correlation in the original environmental study can be attributed to the sample collection methods utilized in that study.

\section{Interferences}

Interferences for the FSM are not well documented. Gold is generally believed to be an excellent collector for mercury, and many believe it sorbs all species of mercury. ${ }^{8}$ Also, previous investigations of this matter indicate that it is possible for gold to become coated with ammonium salts, biogenic waxes, and sulfur compounds. ${ }^{14}$ The Arizona Instruments Corporation notes no interferences for its gold-film mercury vapor analyzer because of the special mallcosorb prefilter, which consists of a mixture of sodium hydroxide and soda lime immediately before the gold film. 
Earlier in our studies, it was suggested that the reason for low recoveries of mercury could be due to the oxidation of the mercury during its deamalgamation by the high-temperature flash. To test this hypothesis, a series of samples were prepared and randomly deamalgamated using air or high-purity nitrogen as the carrier. The inlet to the desorption system was fitted with a T-connector that allowed air to come in one branch of the $\mathrm{T}$ and high-purity nitrogen to come into the other. Prior to flashing the tubes, the Jerome was run several times to flush the system with nitrogen. The use of nitrogen as carrier gas gave no improvement in the method and confirmed that there was no significant oxidation occurring during the deamalgamation process. Furthermore, the trend was for lower recoveries with the nitrogen carrier, although the lowered results were not statistically different.

Soils are highly variable and can contain complex mixtures of primary minerals, secondary clay minerals, iron oxides and hydroxides, chlorides, humic substances, and other components that can greatly affect mercury speciation and sorption. ${ }^{15-17}$ The performance of the adsorbed mercury screening method was not determined for other soil types, and the conditions optimized in this work may not be optimum for different soil types. For other soil types, incubation time and temperature should be optimized prior to using the FSM. Furthermore, the current method should not detect species other than elemental mercury, which is the only form of mercury capable of amalgamating onto the gold-film detector.

During the preliminary evaluation of the FSM, two standards were analyzed to determine its response to them. Even when using a 10-mg sample, the analysis of NIST SRM 8407 resulted in the immediate saturation of the gold-film detector, and no reliable readings could be obtained. This standard was derived from flood plain soils and contains $50 \mu \mathrm{g} / \mathrm{g}$ of mercury that resulted from releases at the Department of Energy's Oak Ridge Y-12 plant. The second standard was an ERA certified quality control standard created by fortifying soil containing high levels of chloride with mercuric nitrate tol.5 mg/kg. The form of mercury in this sample is most probably $\mathrm{HgCl}_{2}$. An analysis of this soil by $\mathrm{CV}$ - 
AA typically yielded results between 1.4 to $1.5 \mathrm{mg} / \mathrm{kg}$, whereas the FSM determinations on $100-\mathrm{mg}$ samples were typically $0.1 \mathrm{mg} / \mathrm{kg}$. The FSM is not sensitive to this form of mercury and it may be that the readings indicated a partial reduction of the mercuric mercury to the elemental state either in situ or during the FSM method.

\section{CONCLUSION}

The adsorbed mercury screening method represents a fast and potentially accurate method for the analysis of mercury in silty clay-type soils, and the results are similar to those obtained by the traditional cold-vapor atomic absorption analysis. The major disadvantage of the method appears to be saturation of the gold film used to detect the mercury. Such a constraint could be minimized by designing dedicated instrumentation or incorporating commercially available, adjustable dilution devices between the final heating unit and the mercury vapor detector. The field screening method may not detect anything other than elemental mercury and, hence, may have a negative bias with certain soil types or environmental situations where the form of mercury is known to be other than elemental. The screening method has been optimized for silty clay materials and it may be unwise to assume that these conditions apply to other materials such as loam soils.

\section{ACKNOWLEDGEMENT}

The authors wish to thank the NASA Environmental Management Division (Code JE) for funding this research. We also wish to acknowledge Microseeps and David J. Masdea (currently of Vaprotech Services, Inc., Valencia, PA) for helpful discussions and technical assistance with the FSM. The $\mathrm{H}$ and $\mathrm{L}$ samples critical to the completion of this project were prepared by Joseph Gorse. Daniel D. White is also acknowledged for assistance with the funding for the project. 
Trade names or manufacturers' names are used in this report for identification only. This usage does not constitute an official endorsement, either expressed or implied, by the National Aeronautics and Space Administration.

\section{REFERENCES}

1. N.S. Bloom and W.F. Fitzgerald, Anal. Chim. Acta, 208, 151 (1988).

2. R. Dumarey, R. Dams, and J. Hoste, Anal. Chem., 57, 2643 (1985).

3. M. Horvat, V. Lupsina, and B. Pihlar, Anal. Chim. Acta, 243, 71 (1991).

4. R. Dumarey, R. Dams, E. Temmerman, and J. Hoste, Anal Chim. Acta, 170, 337 (1985).

5. D.H. Anderson, J.H. Evans, J.J. Murphy, and W.W. White, Anal. Chem., 43, 1511 (1971).

6. W.F. Fitzgerald and G.A. Gill, Anal. Chem., 51, 1714 (1979).

7. Microseeps, Pittsburgh, PA., private communication.

8. N.S. Bloom, Sampling and analysis of mercury in environmental media of importance to the natural gas industry, Gas Research Institute, Chicago, IL. Available through www.gri.org Document Number GRI-94/0033.

9. E. Prestbo, L. Liang, M. Horvat, and N. Bloom, Recent advances in the analytical techniques for the quantification of mercury and mercury compounds in different media, EPA Arsenic and Mercury Workshop on Removal, Recovery, Treatment and Disposal, August 19, 1992. Available through Brooks Rand, Limited, Seattle WA 98107.

10. S.C. Fang, Environ. Sci. and Technol., 12, 285 (1978).

11.U.S. EPA, Test methods for evaluating solid waste, SW-846, Method 7471A (revision 1, 1994). Available through the U.S. Government Printing Office, Washington, DC 20402, Document Number 955-001-00000-1.

12."Mercury use and emission," in Mercury and the Environment, 73-74, Organization for Economic Cooperation and Development, Paris, France (1974). 
13. H.T. Shacklette and J.G. Boerngen, Elemental concentrations in soil and other surficial materials of the conterminous United States, U.S. Geologic Survey, Professional Paper 1270 (1984). Available through the U.S. Government Printing Office, Washington, DC 20402.

14. C. Brosset and A. Iverfeldt, Water, Air, and Soil Pollut., 43, 147 (1989).

15. N.J. Barrow and V.C. Cox, J. Soil Sci., 43, 305 (1992).

16. E. Schuster, Water, Air, and Soil Pollution, 56, 667 (1991).

17. I.I. Skripnichenko and B.N. Zoltareva, Sovict Soil Sci., 15, 49 (1983).

Received: Jariuary 10,2000 Accepted: Februáry 15, 2000 